\title{
EDITORIAL
}

\section{Maternal mortality: changing etiologies will require different approaches}

\section{Journal of Perinatology (2012) 32, 161-162; doi:10.1038/p.2011.143}

Throughout the twentieth century, in the developed world, we observed marked decrease in the risk of maternal mortality due to the common causes of infection, hemorrhage or complications from hypertensive diseases. Antibiotics have successfully treated the common obstetric infections. ${ }^{1}$ Blood banking and the use of uterotonic agents have plummeted the chances of bleeding to death from most obstetric hemorrhage. Routine screening for preeclampsia in the third trimester and then immediate action with anti-hypertensives, magnesium sulfate and induction of labor have dropped the morbidity and mortality related to the hypertensive disorders of pregnancy. All in all, the risk of dying during childbirth has been reduced more than 100-fold over the past 100 years.

Although this has gone on, competing changes have occurred. First, the demographics of pregnant women have changed. As the majority of women now work outside the home, many are choosing to delay child bearing while they establish their careers. This has led to an increased mean maternal age as well as a higher proportion of pregnant women in the advanced maternal age categories of $\geqslant 35$ and $\geqslant 40$ years. These older women are more likely to have medical comorbidities and chronic illnesses. Even more importantly, the obesity epidemic has led to a higher proportion of obese patients and a higher proportion of those women in the highest risk categories of morbidly obese and super obese. Obesity in pregnancy has been associated with pregnancy morbidity, ${ }^{2}$ and it has been demonstrated that obesity is associated with a threefold increased risk of near-miss events. ${ }^{3}$ Thus, the obesity epidemic may contribute to turning around our reduction in maternal mortality.

Another major change is that improved medical care has led to increased numbers of patients with chronic diseases (for example, diabetes, renal disease, cardiac disease, lupus, cystic fibrosis) who can get pregnant. In the 1920s (before the advent of insulin), caring for a patient with pregestational Type 1 diabetes was unheard of; such patients did not survive to become pregnant. This was similar for cystic fibrosis. Now, however, our antepartum services include such patients along with women with end-stage renal disease and severe cardiac disease.

In this issue of the Journal of Perinatology, Burlingame et al. ${ }^{4}$ examined the epidemiology of maternal mortality in Hawaii and determined that the most common cause of pregnancy-related mortality is heart disease. They report a wide range of cardiac disease leading to maternal mortality, including valvular disease, ischemic disease and, of course, cardiomyopathy. This finding is quite striking when one considers statistics from other states. For example, although cardiomyopathy is the leading cause of pregnancy-related death in North Carolina, ${ }^{5}$ its overall cardiomyopathy cause-specific pregnancy-related mortality rate was 0.88 versus Hawaii's fivefold higher rate of 4.48. The authors hypothesize that this extremely high level is likely secondary to overall rises in cardiac disease in Hawaii, which is again likely related to obesity.

Because of the apparent effect on maternal mortality due to maternal comorbidities, such as hypertension, diabetes and obesity, the effort in the United States to reduce maternal mortality will need to focus on screening and management of such conditions. Interestingly, in another paper in this issue Journal of Perinatology by Katsuragi et al., ${ }^{6}$ the authors investigate maternal factors associated with mortality in the setting of dilated cardiomyopathy. In this case series of 29 patients, all 6 with poor prognosis (death or end-stage cardiac disease) were diagnosed in pregnancy. A low left ventricular fractional shortening and diastolic dysfunction were both associated with a poor prognosis as well. Interestingly, although several of the patients demonstrated worsening cardiac function as the pregnancy progressed, of the eight women who opted for termination of pregnancy, there was no progression of their cardiac disease up to 1 year postpartum.

Given that all of the women who were diagnosed before pregnancy did well, this suggests that pre-pregnancy care and stabilization of disease before pregnancy can impact long-term outcomes. Certainly, this has been demonstrated to be true in the case of pregestational diabetes. What we need is a system that encourages primary care providers to consider all women of childbearing age as potential preconception consult patients, and for all obstetric clinicians to refer postpartum women into a primary care practice. However, with an increasingly fragmented health-care system and a great many women only having health insurance during pregnancy, we lack systematic approaches to obtaining such pre-pregnancy or inter-pregnancy care that could lead to improved outcomes and even cost savings.

Perhaps with the emphasis on accountable care organizations in the Affordable Care Act, some of the externalities produced by a lack of preconception and interconceptional care will be 
internalized by the health care system, and organizations will be incentivized to provide such preventive care. The impact that such care might make on diagnosing and treating diabetes and chronic hypertension will likely be enormous. However, there would also be benefits to those patients with downstream morbidities such as cardiac or renal disease. In the end, we have achieved much of the preventable maternal mortality from the historically common causes, but efforts to prevent maternal mortality in the future are going to require comprehensive maternal care before pregnancy, which will involve a broad range of primary care providers.

\section{Conflict of interest}

The author declares no conflict of interest.

Professor AB Caughey Department of Obstetrics and Gynecology, Center for Women's Health, Julie Neupert Stott Director,
Oregon Health and Science University, Portland, OR, USA E-mail:caughey@obsu.edu

\section{References}

1 Caughey AB. Maternal mortality: more than just anecdotal evidence. J Perinatol 2007; 27(10): 595-596.

2 Cnattingius S, Bergstrom R, Lipworth L, Kramer MS. Prepregnancy weight and the risk of adverse pregnancy outcomes. $N$ Engl J Med 1998; 338: 147-152

3 Goffman D, Madden RC, Harrison EA, Merkatz IR, Chazotte C. Predictors of maternal mortality and near-miss maternal morbidity. J Perinatol 2007; 27(10): 597-601.

4 Burlingame J, Horiuchi B, Ohana P, Onaka A, Sauvage LM. The contribution of heart disease to pregnancy-related mortality according to the pregnancy mortality surveillance system. J Perinatol 2012; 32: 163-169.

5 Berg CJ, Harper MA, Atkinson SM, Bell EA, Brown HL, Hage ML et al. Preventability of pregnancy-related deaths: results of a state-wide review. Obstet Gynecol 2005; 106 $1228-1234$

6 Katsuragi S, Omoto A, Kamiya C, Ueda K, Sasaki Y, Yamanaka K et al. Risk factors for maternal outcome in pregnancy complicated with dilated cardiomyopathy. J Perinatol 2012; 32: 170-175. 